Results There were 57 presentations of 34 young persons identified. The mean age was 13.4 years, with a female to male ratio of $8: 2$. A progressive increase in the proportion of presentations in the 0-12 years cohort was identified. 25\% presentations were unknown to Child and Adolescent Mental Health Services (CAMHS) at the time of presentation. Anorexia Nervosa Restricting type accounted for the vast majority of presentations (68\%), followed by ARFID (14\%). 54 out of 57 presentations required admission, and the mean length of admission was 12 days (range 1-41). The average median $\%$ BMI on admission was $72 \%$ and $80 \%$ at discharge. Nearly half of those admitted were discharged to a CAMHS inpatient unit. Evidence of compliance of recording JMARSIPAN parameters was approximately $60 \%$ on admission and $43 \%$ on discharge.

Conclusion This study provides important data regarding patterns of clinical presentation for this high risk population, and will be useful in service planning and development. It confirms an increase in presentations of young persons with restrictive eating disorders over the last 5 years, in particular in the age $0-12$ cohort, in line with international trends. Young people are presenting with severe physical complications, and the majority required admission for medical stabilization. In line with services internationally, the study indicates historic deficits in the standards of risk assessment compared to JMARSIPAN guidelines. The Tallaght University Hospital has introduced new Paediatric Guidelines on Management of Anorexia Nervosa in 2019, and we will complete the audit of practice in one year.

\section{GP94 PAEDIATRIC RADIATION: DO WE KNOW WHAT WE ARE DOING?}

${ }^{1}$ Niamh Ryan*, ${ }^{2}$ Deirdre Rafferty. ${ }^{1}$ Mercy University Hospital, cork, Ireland; ${ }^{2}$ Mercy University Hospital, Cork, Ireland

\subsection{6/archdischild-2019-epa.159}

Radiation is a commonly used diagnostic tool in paediatric medicine. We know that paediatric patients will have a longer lifetime risk of radiation sequelae. Therefore, the judicious use of radiation should be paramount for practitioners. In a cross section of paediatric staff in a secondary level paediatric centre, we examined aspects of their radiation knowledge by questionnaire.

The objectives of this study were;

1. To determine radiation dose awareness

2. To assess knowledge of the correct modality in defined clinical scenarios

3. To evaluate the perceived dosing equivalent of a number of commonly ordered modalities, examining the accuracy

This supervised questionnaire was completed by a range of paediatric staff, both medical and nursing. Immediate feedback was given at the end of the questionnaire.

Despite utilising radiation for children on a daily basis the knowledge of risk was suboptimal. In certain clinical scenarios where no modality was required, up to $33 \%$ would have performed an unjustified investigation. Underestimation of radiation dose was as high as $93 \%$ in one modality, whilst $40 \%$ of those surveyed thought that ultrasound was associated with radiation exposure.
Our results highlight the need for further radiation protection teaching. Participants were given immediate feedback on completing the questionnaire, however further efforts are needed to ensure that practitioners have the knowledge to weigh the risks and benefits of appropriate medical imaging for their patients.

\section{GP95 A PROSPECTIVE AUDIT OF PAEDIATRIC ATTENDANCES AT A REGIONAL GENERAL EMERGENCY DEPARTMENT AND PAEDIATRIC ASSESSMENT UNIT}

Kevin Gaughan*, Sean Casey, Alan Finan. Paediatric Department, Cavan General Hospital, Cavan, Ireland

\subsection{6/archdischild-2019-epa.160}

Introduction Cavan General Hospital's Paediatric Assessment Unit (PAU) was the first of its' kind in the Republic of Ireland. The PAU was opened in 2011 with the aim of addressing the growing number of urgent paediatric attendances. It deals with all urgent medical and surgical cases and provides some scheduled care for routine procedures. Published studies have indicated that PAU-type units can improve efficiency, reduce admission rates and overall costs.

Aim The PAU's Operational Policy mandates regular monitoring of activity relative to a variety of key performance indicators. The aim of this audit was to review and contrast ED/ PAU activity in order to assess the impact of the PAU. Performance standards were based on the ICTS's recommended wait-times and the RCEM's four-hour standard for outcome decision.

Methods Overall trends in paediatric emergency activity over the period from 2009 to 2018 were reviewed. A threemonth prospective audit was conducted of all attendees of CGH's ED and PAU less than 16 years old from the 1st of September to the 30th November of 2018. The key performance indicators assessed were time to triage, compliance with recommended wait-times, admission rates and compliance with the four-hour standard. Results were compared with those of a similar audit conducted over the same season in 2009. Statistical analysis was performed using Microsoft Excel 2010.

Results Between 2013 and 2018 urgent attendances increased by $46.8 \%$ from 5,487 to 8,059 . During this period PAU activity increased by $13.4 \%$ yet overall paediatric admissions rates fell from $24.3 \%$ to $17.9 \%$. Over the three-month audit period 1,764 attendances were recorded. Compared to 2009 more patients in 2018 were triaged within 15 minutes of arrival $(60 \%$ vs $45 \%, \mathrm{P}<0.001)$ and more were seen within 1 hour of triage $(80 \%$ vs $75 \%, \mathrm{p}<0.01)$. In comparison to ED attendees, those attending the PAU were more likely to be triaged within 15 minutes (69\% vs. 47\%, p<0.001), had higher compliance to recommended wait-times $(89 \%$ vs. $65 \%, \mathrm{p}<0.001)$, had lower rates of admissions $(21 \%$ vs. $26 \%, p<0.05)$ and had higher compliance to the four-hour standard (94\% vs. $88 \%, \mathrm{p}<0.001)$.

Conclusions Urgent paediatric referral numbers in our region have shown a significant increase between the years 2013 to 2018. However the percentage of these referrals admitted to hospital has reduced. Since the PAU has opened there has been an improvement in key performance indicators and there is, at present, better compliance to accepted standards. 